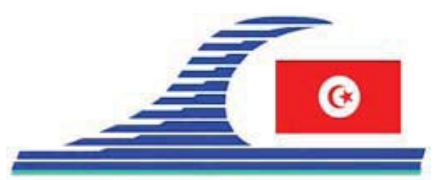

Conférence Méditerranéenne Côtière et Maritime EDITION 1, HAMMAMET, TUNISIE (2009)

Coastal and Maritime Mediterranean Conference

Disponible en ligne - http://www.paralia.fr-Available online

\title{
Un système d'ancrage subaquatique alternatif au béton
}

\section{Société NAUTISCAPHE ${ }^{1}$}

1. NAUTISCAPHE : Nautisme, Ancrages \& Arrimages Subaquatiques, 2375 Avenue Président John F. Kennedy, 83140 Six-Fours-les-Plages, France. http://www.nautiscaphe.com/ info.nautiscaphe@gmail.com

\section{Résumé :}

Nautiscaphe en seulement 3 ans d'existence a :

- créé et développé un système d'ancrage subaquatique alternatif au béton,

- su convaincre les plaisanciers de l'efficacité de ces ancrages dénommés «Ellipses» environnementaux, sécuritaires et économiques,

- conçu à partir des ancrages de type «Ellipses » des extensions portuaires pour différentes communes en Méditerranée et en Atlantique, soit plus de 1050 places supplémentaires,

- commercialisé, aux vues des besoins, des fournitures portuaires (chaîne, cordage, manilles, bouées....).

Afin de répondre le plus efficacement possible aux besoins des clients, nous avons structuré la société Nautiscaphe en alliant: bureaux d'études, bureaux des affaires, bureaux commerciaux, bureaux d'exploitations.

Durant ces 3 ans, l'innovation et l'utilisation de notre gamme d'ancrages « Ellipse » ont été récompensées par:

- Le prix du RDT (réseau de développement technologique) PACA, prix parrainé par VERITAS pour la partie managériale et accompagné sur nos chantiers pour la partie certification de la sécurité de nos ancrages.

- Réseau Entreprendre PACA a fait lauréat Nautiscaphe à l'unanimité devant un jury de 15 entrepreneurs.

- Le Pôle Mer PACA a soutenu le projet Ellipse Nautiscaphe, et deux modules de solutions d'éco mouillage et brise clapot viennent d'être déposés pour labellisation.

\section{Mots-clés :}

Ancrage - Ellipse - Eco mouillage - Brise clapot - Nautiscaphe

\section{NAUTISCAPHE}




\section{Type d'ancrage Ellipse}

Notre produit est le fruit de plus de 24 mois d'études et de tests divers pour aboutir à un brevet international, voir figure 1. Il peut s'insérer dans tous les sédiments : sable, vase, matte morte de posidonie, posidonie et même les substrats caillouteux.
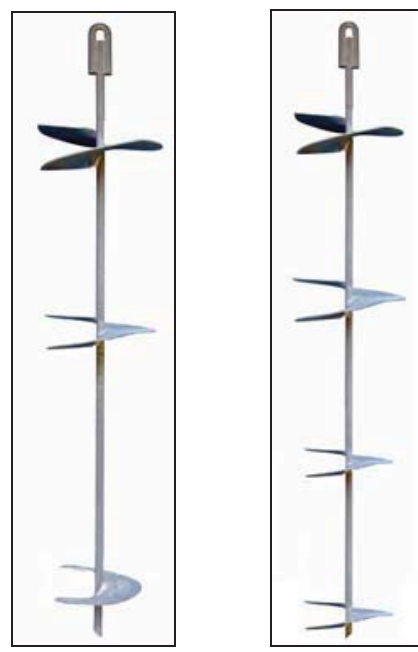

Figure 1. Ancrage de type Ellipse.

\section{Evolution}

Depuis le mois de juillet 2009 la société Nautiscaphe a signé un partenariat commercial avec la société Poralu Marine, afin de réunir l'efficacité en surface et subaquatique pour les marinas.

A ce jour, notre panel d'activité, autour des travaux maritimes, évolue et concerne :

- Les études et conceptions d'ancrage pour tout type de structures flottantes ou immergées (figure 2),

- La maîtrise d'ouvrage d'ancrage pour tout type de structures flottantes ou immergées (figure 3),

- L'expertise portuaire,

- La protection anti-pollution.

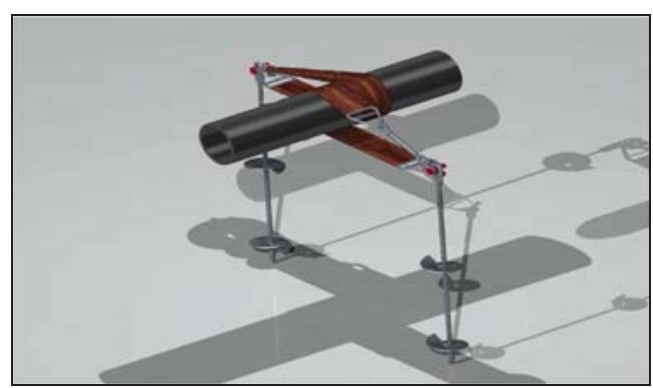

Figure 2. Ancrage pipe.

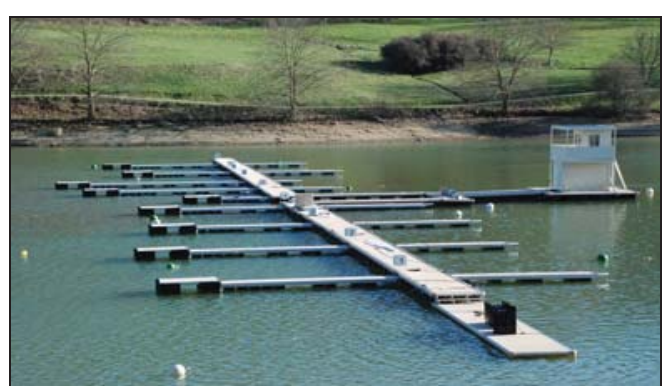

Figure 3. Ancrage ponton championnat du monde junior d'aviron. 


\section{Le principe d'ancrage de mouillage}

Ellipse, une bouée subsurface moussée en haute densité et une chaîne de lest, permettent d'amortir les différents chocs et déformations structurelles que subit l'unité flottante à cause du vent et de la houle, la tension du mouillage ne s'effectue plus directement sur le point d'ancrage. Cette bouée est importante car il n'y a plus de raguage sur les fonds marins (figure 4).
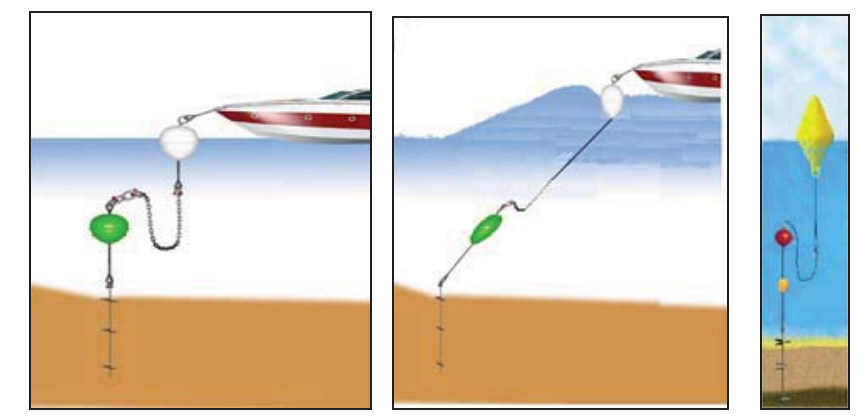

Figure 4. Principe d'ancrage mouillage et balisage.

\section{Description et situation du produit}

- L'ancre est composée d'une tige sur laquelle sont soudées des ellipses.

- Les ellipses sont ancrées dans le sol.

\section{Effort et déplacements}

- L'effort considéré est celui de l'arrachement de l'ancrage.

- Pour le calcul dont les résultats sont montrés sur la figure 5, l'ancre est verticale et l'effort qui lui est appliqué est également vertical.

- Comme l'ancre est soumise uniquement à un effort vertical, on peut considérer que la tige représente une liaison pivot glissant.

- La tige est également arrêtée en translation (verticalement).

- Une pression est appliquée sur les ellipses pour valider l'ensemble de la tenue de l'ancre dans le sol.

- Les deux matériaux sont soudés, il faut donc prévoir une perte de résistance $(0,8)$ dans la zone affectée thermiquement (ZAT).

- La contrainte doit être inférieure à $272 \mathrm{MPa}$ pour les ellipses et $480 \mathrm{MPa}$ pour la tige.

- La contrainte dans l'ancre est inférieure à la contrainte maximum admissible par les différents matériaux.

\section{Réalisations}

2007-2008: Réalisation d'une zone de mouillage organisé sur une Zone Natura 2000 au Brusc dans le Var soit : 164 places, voir figure 6.

2008-2009: Extension portuaire de 795 places sur Arcachon atlantique dont les essais ont été réalisés avec VERITAS, voir figure 7. 
2009 : Expertise et refonte portuaire du port Napoléon à Port St Louis du Rhône, dans les Bouches du Rhône.

2009: Etudes et conceptions des filières conchylicoles dont les essais ont été effectués avec VERITAS (figures 8 et 9).

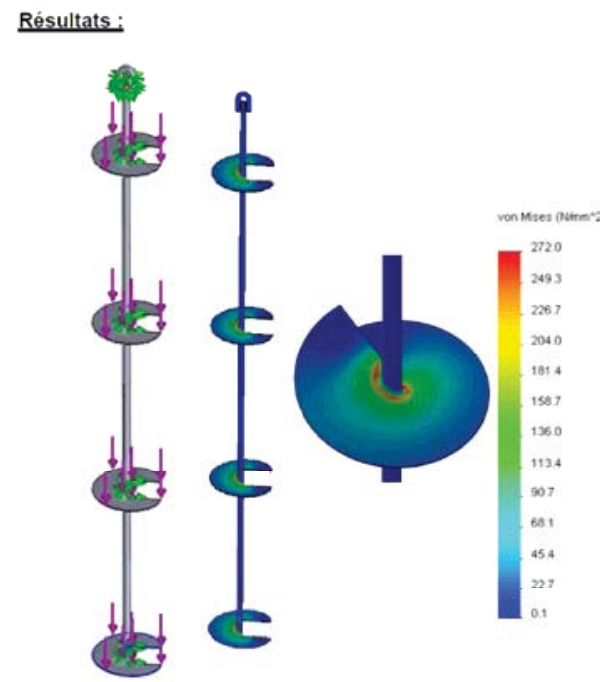

Figure 5. Modélisation

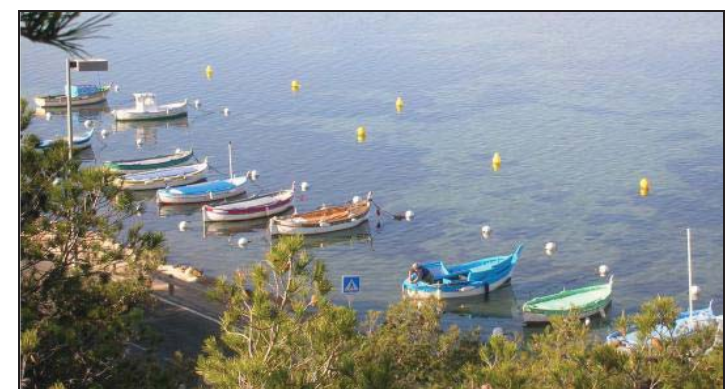

Figure 6. Mouillages (164) au Brusc dans le Var.

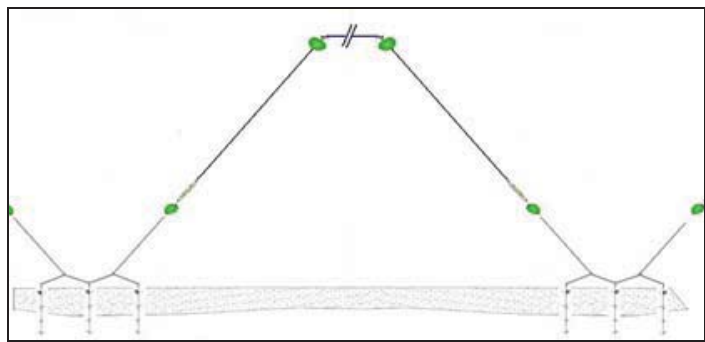

Figure 8. Dessin pour étude filières.

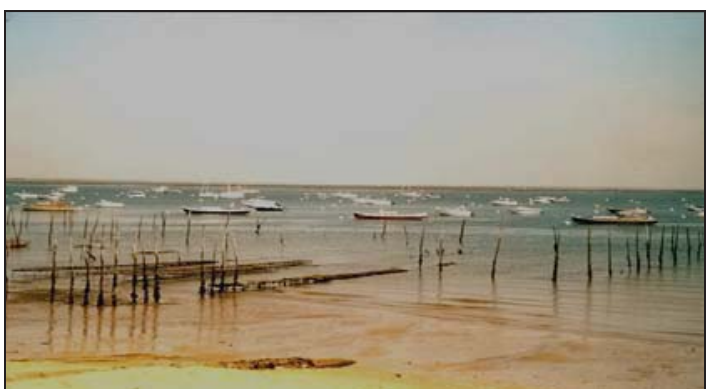

Figure 7. Extension portuaire de 795 places à Arcachon (Gironde).

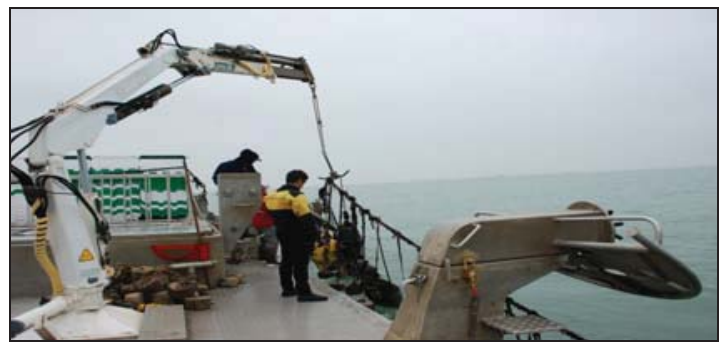

Figure 9. Etudes filières conchylicoles.

\section{Conclusion}

L'ancrage de type Ellipse de la société Nautiscaphe résiste à un effort d'arrachement vertical. Il est adapté à la plupart des sédiments meubles. 